\title{
Research funding acknowledgments
}

Research funding over the last 8 years, with scientific results that found their way into this monograph, is gratefully acknowledged:

1. German Priority Program 1748 (DFG SPP 1748) https://www.uni-due.de/spp1748/ with the project Structure Preserving Adaptive Enriched Galerkin Methods for Pressure-Driven 3D Fracture Phase-Field Models https://www.uni-due.de/spp1748/adaptive_enriched_galerkin_methods

2. FWF (Austrian Science Fund) project P-29181 Goal-oriented error control for phasefield fracture coupled to multiphysics problems http://www.numa.uni-linz.ac.at/Research/Projects/P29181/index.shtml

3. German Priority Program 1962 (DFG SPP 1962)

https://spp1962.wias-berlin.de/

with project No.15 Optimizing Fracture Propagation Using a Phase-Field Approach https://spp1962.wias-berlin.de/project.php?projectID=15

4. Member in the Cluster of Excellence PhoenixD (Photonics, Optics, and Engineering - Innovation Across Disciplines) (EXC 2122)

https://www.phoenixd.uni-hannover.de/en/

5. DAAD (German Academic Exchange Service) Indo-German Higher Education Partnership 2019-2023, Leibniz University Hannover - IIT Indore/Germany - India

6. Alexander von Humboldt Foundation ${ }^{11}$ Feodor Lynen Research Fellowship (20132014)

https://www.oden.utexas.edu/about/news/228/

7. ICES ${ }^{12}$ Post-doctoral fellowship ${ }^{13}$ (2013):

https://www.oden.utexas.edu/media/uploaded-images/351.jpg

11 https://www.humboldt-foundation.de/web/home.html

12 https://www.oden.utexas.edu/

13 https://www.oden.utexas.edu/programs/postdoc/ 
